\title{
inu \\ Novel Antifungal Dimers from the Roots of Taiwania cryptomerioides
}

\author{
Ming-Jen Cheng ${ }^{1,+}{ }^{\oplus}$, Ming-Der $\mathrm{Wu}^{1}{ }^{1}$, Chao-Lin Chang ${ }^{2}$, Hsun-Shuo Chang ${ }^{3} \mathbb{C}$, Chiou-Fung Chyu ${ }^{4,+}$ \\ and Yueh-Hsiung Kuo $5,6,7, *$ (1)
}

1 Bioresource Collection and Research Center (BCRC), Food Industry Research and Development Institute (FIRDI), Hsinchu 300, Taiwan; chengfirdi@gmail.com (M.-J.C.); wmd@firdi.org.tw (M.-D.W.)

2 Product \& Process Research Center (PPRC), Food Industry Research and Development Institute (FIRDI), Hsinchu 300, Taiwan; ccl@firdi.org.tw

3 School of Pharmacy, College of Pharmacy, Kaohsiung Medical University, Kaohsiung 807, Taiwan; hschang@kmu.edu.tw

4 Department of Chemistry, National Taiwan University, Taipei 106, Taiwan; cmj@firdi.org.tw

5 Department of Biotechnology, Asia University, Taichung 413, Taiwan

6 Department of Chinese Pharmaceutical Sciences and Chinese Medicine Resources, College of Pharmacy, China Medical University, Taichung 404, Taiwan

7 Chinese Medicine Research Center, China Medical University, Taichung 404, Taiwan

* Correspondence: kuoyh@mail.cmu.edu.tw

+ These authors contributed equally to this work.

Citation: Cheng, M.-J.; Wu, M.-D.; Chang, C.-L.; Chang, H.-S.; Chyu, C.-F.; Kuo, Y.-H. Novel Antifungal Dimers from the Roots of Taiwania cryptomerioides. Molecules 2022, 27, 437. https://doi.org/10.3390/ molecules27020437

Academic Editor: Akihito Yokosuka

Received: 6 December 2021

Accepted: 14 December 2021

Published: 10 January 2022

Corrected: 16 September 2022

Publisher's Note: MDPI stays neutral with regard to jurisdictional claims in published maps and institutional affiliations.

Copyright: (C) 2022 by the authors. Licensee MDPI, Basel, Switzerland. This article is an open access article distributed under the terms and conditions of the Creative Commons Attribution (CC BY) license (https:// creativecommons.org/licenses/by/ $4.0 /)$.

\begin{abstract}
Five new dimer compounds, namely Taiwaniacryptodimers A-E (1-5), were isolated from the methanol extract of the roots of Taiwania cryptomerioides. Their structures were established by mean of spectroscopic analysis and comparison of NMR data with those of known analogues. Their antifungal activities were also evaluated. Our results indicated that metabolites 1, 2, 4, and 5 displayed moderate antifungal activities against Aspergillus niger, Penicillium italicum, Candida albicans, and Saccharomyces cerevisiae.
\end{abstract}

Keywords: Taiwania cryptomerioides; Taxodiaceae; novel skeleton; dimer; diterpenoid; antifungal activities

\section{Introduction}

Taiwan cedar (Taiwania cryptomerioides Hayata), also known as Asia cedar, is an essential economic tree species native to Taiwan. It belongs to the same Mesozoic and Cenozoic Tertiary relics with Ginkgo biloba, Sequoiadendron giganteum, and Metasequoia glyptostroboides. It is a rare and precious tree species [1]. It belongs to the gymnosperm phylum, Coniferae, Coniferales, Taxodiaceae, Taiwania, one genus and one species. In 1904, the Japanese Prof. S. Konisi was first discovered in Nantou County, Taiwan. It was named by the botanist Prof. Hayata Bunzo in 1906 and named after Taiwan as its genus, "Taiwania". The meaning of the species name "cryptomerioides" is "like Cryptomeria". It can be seen that the morphological characteristics of Taiwan fir is similar to Cryptomeria japonica (L. f) D. Don. As far as the nature of the wood is concerned, Taiwan cedar is straight, delicate, and beautiful, with a light and soft texture, easy to process, and its heartwood has a beautiful yellow-red texture, which is quite distinctive. Weather resistance, decay resistance, and ant resistance are similar to those of cypress. It is a material with superior properties and is often used for construction, furniture, door panels, and thin panels. The fresh inner skin of Taiwan fir is mashed to apply it to poisonous snake bites. The leaves also have the effects of mashing juice and involving poison and are used for anti-inflammatory, diuretic, and gonorrhea treatment.

Taiwania cryptomerioides (Taxodiaceae) is taxonomically included in one genus and one species of endemic plants in Taiwan. It contains more than $6 \%$ of essential oil in its 
heartwood [1]. T. cryptomerioides is a vital building material with high value in Taiwan. Earlier phytochemical investigations of this plant mainly focused on its heartwood [2-4] and bark constituents [5-9] have also been found. In the past, interesting compounds isolated from the heartwood and bark of T. cryptomerioides and those with biological activity prompted us to study the chemical composition of their roots. Several kinds of sesquiterpenes with unique and novel structures have also been obtained from its roots [10-14]. However, compared with the composition of different parts of T. cryptomerioides in the past literature, it is found that there is very little research on the roots, and it is worth continuing to explore its composition. In this paper, we would like to report the five new dimers, namely, Taiwaniacryptodimers A-E (1-5), isolated from the roots of the T. cryptomerioides. The $\mathrm{MeOH}$ extract of the roots of $T$. cryptomerioides was suspended in $\mathrm{H}_{2} \mathrm{O}$ and partitioned between $\mathrm{H}_{2} \mathrm{O}$ and EtOAc. The EtOAc-soluble portion was subjected to repeated silica gel column chromatography and semipreparative normal phase-HPLC to afford compounds 1-5. Herein, the isolation and structure elucidation of five new compounds and their antifungal activity is described.

\section{Results and Discussion}

\subsection{Structure Elucidation of Compounds}

Compound 1 was isolated as gum with a specific rotation $[\alpha]_{\mathrm{D}}^{30}=+131.7^{\circ}(c 0.09$, $\mathrm{CHCl}_{3}$ ). The HR-EI-MS of 1 gave a molecular ion at $m / z 666.2828$, establishing the molecular formula of 1 as $\mathrm{C}_{40} \mathrm{H}_{42} \mathrm{O}_{9}$, with 20 degrees of unsaturation. The EI-MS fragmental ions of 1 at $m / z 350\left[\mathrm{C}_{20} \mathrm{H}_{14} \mathrm{O}_{6}\right]^{+}$and $316\left[\mathrm{C}_{20} \mathrm{H}_{28} \mathrm{O}_{3}\right]^{+}$, as well as forty carbon signals found in the ${ }^{13} \mathrm{C}$ NMR spectrum hinted that 1 was a dimeric diterpenoid (Figure 1 ). The UV maxima at 229,284 , and $314 \mathrm{~nm}$ suggested the presence of a benzoyl functionality. The absorption bands for hydroxyl $\left(3582 \mathrm{~cm}^{-1}\right), \gamma$-lactone $\left(1791 \mathrm{~cm}^{-1}\right)$, and aromatic $\left(1616,1499 \mathrm{~cm}^{-1}\right)$ groups were observed in its IR spectrum. The ${ }^{1} \mathrm{H}$ and ${ }^{13} \mathrm{C}$ NMR data of 1 (Tables 1 and 2) exhibited signals for constituent upper monomer- 1 (A): two tertiary methyl groups $\left[\delta_{\mathrm{H}} 0.91\right.$ and 0.88 (each $3 \mathrm{H}, \mathrm{s}, \mathrm{Me}-18$, and Me-19], an isopropyl group attached to a phenyl group $\left[\delta_{\mathrm{H}} 1.20(3 \mathrm{H}, \mathrm{d}, J=6,8 \mathrm{~Hz}, \mathrm{Me}-16), 1.24(3 \mathrm{H}, \mathrm{d}, J=6.8 \mathrm{~Hz}, \mathrm{Me}-17)\right.$ and $3.25(1 \mathrm{H}$, sept, $J=6.8$ $\mathrm{Hz}, \mathrm{H}-14)]$, one aromatic proton $\left[\delta_{\mathrm{H}} 6.69(1 \mathrm{H}, \mathrm{s}, \mathrm{H}-11)\right]$, and a typical downshifted $\mathrm{H}_{\beta}-1$ signal of a dehydroabietane diterpene $\left[\delta_{\mathrm{H}} 1.77(1 \mathrm{H}, \mathrm{m})\right]$; a two set contiguous structural sequence was derived from COSY correlations $\left[\delta_{\mathrm{H}} 1.50 / 1.77\right.$ (each $\left.1 \mathrm{H}, \mathrm{m}, \mathrm{CH}_{2}-1\right), 1.40 / 1.77$ (each $1 \mathrm{H}, \mathrm{m}, \mathrm{CH}_{2}-2$ ), 1.23/1.38 (each $\left.1 \mathrm{H}, \mathrm{m}, \mathrm{CH}_{2}-3\right) ; 1.30(1 \mathrm{H}, \mathrm{m}, \mathrm{H}-5)$ ) 1.80/1.96 (each $1 \mathrm{H}$, $\left.\mathrm{m}, \mathrm{CH}_{2}-6\right)$ ], and a nonequivalent $\mathrm{CH}_{2}$ was observed [ $\delta_{\mathrm{H}} 2.50 / 3.29$ (each $1 \mathrm{H}, \mathrm{d}, J=14.4 \mathrm{~Hz}$, $\left.\mathrm{CH}_{2}-20\right]$. Those data proved that the structure of constituent upper monomer- 1 was related to demethylsalvicanol [15]. The ${ }^{1} \mathrm{H}$ and ${ }^{13} \mathrm{C}$ NMR data of 1 (Table 1) also exhibited another set of signals for constituent lower monomer-2 (B): two methylenedioxy groups $\left(\delta_{\mathrm{H}} 5.94\right.$ $\left(2 \mathrm{H}, \mathrm{s}, \mathrm{C}^{\prime}, 5^{\prime}-\mathrm{OCH}_{2} \mathrm{O}-\right), 5.98 / 6.00$ (each $1 \mathrm{H}, J=1.6 \mathrm{~Hz}, \mathrm{C}^{\prime \prime}, 5^{\prime \prime}-\mathrm{OCH}_{2} \mathrm{O}-$ ) at two benzene moieties, respectively, and $\mathrm{CH}_{2}$ protons in a $\gamma$-position of a $\gamma$-lactone ring $\left(\delta_{\mathrm{H}} 3.94(1 \mathrm{H}\right.$, $\left.\mathrm{dd}, J=13.2,2.8 \mathrm{~Hz}, \mathrm{H}-9^{\prime \prime}\right), 4.81\left(1 \mathrm{H}, \mathrm{dd}, J=13.2,2.8 \mathrm{~Hz}, \mathrm{H}-9^{\prime \prime}\right)$. Additionally, two ABX systems $(6 \mathrm{H})$ of aromatic protons were observed $\left(\delta_{\mathrm{H}} 6.91\left(1 \mathrm{H}, \mathrm{d}, J=8.0 \mathrm{~Hz}, \mathrm{H}-2^{\prime}\right), 6.94(1 \mathrm{H}\right.$, s, H-6 $\left.{ }^{\prime}\right), 6.80\left(1 \mathrm{H}, \mathrm{d}, J=8.0 \mathrm{~Hz}, \mathrm{H}-3^{\prime}\right)$, and $\delta_{\mathrm{H}} 6.42\left(1 \mathrm{H}, \mathrm{s}, \mathrm{H}-6^{\prime \prime}\right), 6.44\left(1 \mathrm{H}, \mathrm{d}, J=8.0, \mathrm{H}-2^{\prime \prime}\right)$, $\left.6.73\left(1 \mathrm{H}, \mathrm{d}, J=8.0 \mathrm{~Hz}, \mathrm{H}-3^{\prime \prime}\right)\right)$, besides two benzylic protons $\left(\delta_{\mathrm{H}} 5.06\left(1 \mathrm{H}, \mathrm{s}, \mathrm{H}-7^{\prime}\right), 6.47(1 \mathrm{H}\right.$, $\left.\left.\mathrm{s}, \mathrm{H}-\mathrm{7}^{\prime \prime}\right)\right)$. The ${ }^{13} \mathrm{C}-\mathrm{NMR}$ spectrum and DEPT experiment showed 20 signals including a $\gamma$-lactone carbonyl C-atom $\left(\delta_{\mathrm{C}} 171.7\left(\mathrm{C}-9^{\prime}\right)\right)$, twelve aromatic $\mathrm{C}$-atoms, and three $\mathrm{CH}_{2}\left(\delta_{\mathrm{C}}\right.$ $\left.101.4\left(\mathrm{C}^{\prime}, 5^{\prime}-\mathrm{OCH}_{2} \mathrm{O}-\right), 101.4\left(\mathrm{C}^{\prime \prime}, 5^{\prime \prime}-\mathrm{OCH}_{2} \mathrm{O}-\right), 69.6\left(\mathrm{C}-9^{\prime \prime}\right)\right)$ and two $\mathrm{CH}$ groups $\left(\delta_{\mathrm{C}} 77.4\right.$ $\left.\left(\mathrm{C}-7^{\prime}\right), 128.7\left(\mathrm{C}-7^{\prime \prime}\right)\right)$. The above NMR spectroscopic data of constituent lower monomer-2 are very similar to that of Taiwanin A [16], suggesting constituent lower monomer-2 was a lignanoid-derivative. Thus, the gross structure of $\mathbf{1}$ is composed of demethylsalvicanol and Taiwanin A. After calculating the degree of unsaturation, subtracting the above-inferred part, there is still one degree of unsaturation. The C- $7^{\prime} / C-12$ and C- $8^{\prime}$ and C-11 linkage of the two monomers in $\mathbf{1}$ was through a $\mathrm{O}$-linkage functionality; judged by calculating the degree of unsaturation, subtracting the above inferred parts $A$ and $B$, there is still one degree 
of unsaturation. Its molecular formula contains nine oxygen atoms and the downfield shift of $\mathrm{H}-7^{\prime}\left(\delta_{\mathrm{H}} 5.06\right)$ in constituent monomer-1, compared to that of the Taiwanin A analogues.
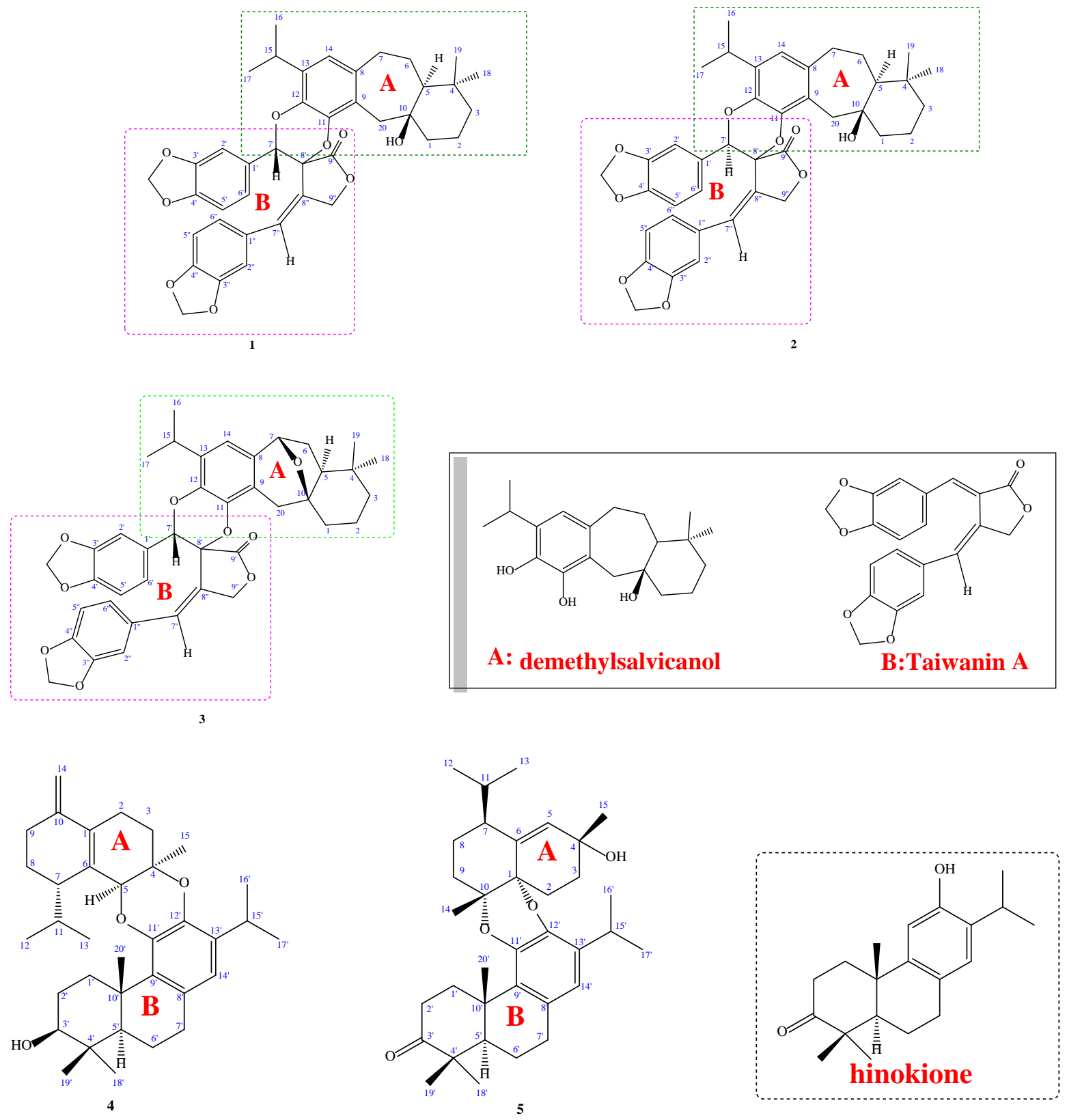

Figure 1. Compounds 1-5, isolated from the roots of Taiwania cryptomerioides.

Compound 2 is also a gum with a specific rotation $[\alpha]_{\mathrm{D}}^{30}=-26.2\left(c 0.08, \mathrm{CHCl}_{3}\right)$. The HR-EI-MS of 2 also showed a molecular ion at $m / z$ 666.2823, which corresponded to the molecular formula, $\mathrm{C}_{40} \mathrm{H}_{42} \mathrm{O}_{9}$, indicating 20 degrees of unsaturation. The EI-MS fragmental ions of 2 at at $m / z 350\left[\mathrm{C}_{20} \mathrm{H}_{14} \mathrm{O}_{6}\right]^{+}$and $316\left[\mathrm{C}_{20} \mathrm{H}_{28} \mathrm{O}_{3}\right]^{+}$, indicated that 2 was also a dimeric diterpenoid. The IR spectrum indicated the presence of hydroxyl $\left(3576 \mathrm{~cm}^{-1}\right)$, ester carbonyl $\left(1791 \mathrm{~cm}^{-1}\right)$, and aromatic $\left(1616,1499,1447 \mathrm{~cm}^{-1}\right)$ groups. The UV maxima at 229, 284, and $314 \mathrm{~nm}$ indicated a benzoyl functionality. Comparison of ${ }^{1} \mathrm{H}$ and ${ }^{13} \mathrm{C}$ NMR data of 1 and $\mathbf{2}$ (Tables 1 and 2) showed that the signals of constituent upper monomer- 1 of $\mathbf{2}$ were almost the same as those of $\mathbf{1}$; thus, the structure of constituents upper monomer-1 and lower monomer-2 were related to demethylsalvicanol and Taiwanin A, respectively. 
Table 1. ${ }^{1} \mathrm{H}$ NMR data for compounds $1-5$ in $\mathrm{CDCl}_{3}\left(\delta\right.$ in ppm, $J$ in $\mathrm{Hz}, 500 \mathrm{MHz}$ in $\left.\mathrm{CDCl}_{3}\right)$.

\begin{tabular}{|c|c|c|c|c|c|}
\hline No & 1 & 2 & 3 & 4 & 5 \\
\hline \multicolumn{6}{|l|}{ Part A } \\
\hline 1 & $\begin{array}{l}1.50(\mathrm{~m}) \\
1.77(\mathrm{~m})\end{array}$ & $\begin{array}{l}1.36(\mathrm{~m}) \\
1.72(\mathrm{~m})\end{array}$ & $\begin{array}{l}1.78(\mathrm{~m}) \\
1.96(\mathrm{~m})\end{array}$ & & \\
\hline 2 & $\begin{array}{l}1.40(\mathrm{~m}) \\
1.77(\mathrm{~m})\end{array}$ & $\begin{array}{l}1.37(\mathrm{~m}), \\
1.70(\mathrm{~m})\end{array}$ & $\begin{array}{l}1.57(\mathrm{~m}), \\
1.79(\mathrm{~m})\end{array}$ & $\begin{array}{l}2.20(\mathrm{~m}) \\
2.40(\mathrm{~m})\end{array}$ & $1.81(\mathrm{~m})$ \\
\hline 3 & $\begin{array}{l}1.23(\mathrm{~m}) \\
1.38(\mathrm{~m})\end{array}$ & $\begin{array}{l}1.23(\mathrm{~m}) \\
1.49(\mathrm{~m})\end{array}$ & $\begin{array}{l}1.50(\mathrm{~m}) \\
1.11(\mathrm{~m})\end{array}$ & $\begin{array}{c}1.62(\mathrm{dt}, J=13.2,6.0) \\
2.04(\mathrm{dt}, J=13.2 \\
6.0)\end{array}$ & $\begin{array}{l}1.70(\mathrm{~m}) \\
2.81(\mathrm{~m})\end{array}$ \\
\hline $\begin{array}{l}4 \\
5\end{array}$ & $1.30(\mathrm{~m})$ & $1.28(\mathrm{~m})$ & $1.80(\mathrm{~m})$ & $4.28(\mathrm{~s})$ & $5.65(\mathrm{~d}, I=2.0)$ \\
\hline 6 & $\begin{array}{l}1.80(\mathrm{~m}) \\
1.96(\mathrm{~m})\end{array}$ & $\begin{array}{l}1.80(\mathrm{~m}) \\
1.99(\mathrm{~m})\end{array}$ & $\begin{array}{l}1.91(\mathrm{~m}) \\
2.12(\mathrm{~m})\end{array}$ & & \\
\hline 7 & $\begin{array}{l}2.69(\mathrm{~m}) \\
2.79(\mathrm{~m})\end{array}$ & $\begin{array}{l}2.70(\mathrm{~m}) \\
2.77(\mathrm{~m})\end{array}$ & $\begin{array}{c}4.89(\text { br. d, J }=6.4), \\
2.77(\mathrm{~m})\end{array}$ & $2.40(\mathrm{~m})$ & $\begin{array}{l}2.22(\mathrm{br} \mathrm{d} \\
J=13.2)\end{array}$ \\
\hline $\begin{array}{c}8 \\
9 \\
10\end{array}$ & & & & $\begin{array}{l}1.25(\mathrm{~m}) \\
1.51(\mathrm{~m}) \\
2.20(\mathrm{~m}) \\
2.40(\mathrm{~m})\end{array}$ & $\begin{array}{l}1.80(\mathrm{~m}) \\
1.65(\mathrm{~m}) \\
1.41(\mathrm{~m}) \\
1.74(\mathrm{~m})\end{array}$ \\
\hline $\begin{array}{l}10 \\
11 \\
12 \\
13\end{array}$ & & & & $\begin{array}{c}2.28(\mathrm{~m}) \\
0.94(\mathrm{~d}, J=6.8) \\
0.75(\mathrm{~d}, J=6.8)\end{array}$ & $\begin{array}{c}2.12(\mathrm{~m}) \\
0.84(\mathrm{~d}, J=6.8) \\
0.94(\mathrm{~d}, J=6.8)\end{array}$ \\
\hline 14 & $6.69(\mathrm{~s})$ & $6.64(\mathrm{~s})$ & $6.60(\mathrm{~s})$ & $\begin{array}{l}4.77(\mathrm{~s}) \\
4.93(\mathrm{~s})\end{array}$ & $1.30(\mathrm{~s})$ \\
\hline $\begin{array}{l}15 \\
16 \\
17 \\
18 \\
19 \\
20\end{array}$ & $\begin{array}{c}3.25(\text { sept }, J=6.8) \\
1.20(\mathrm{~d}, J=6.8) \\
1.24(\mathrm{~d}, J=6.8) \\
0.88(\mathrm{~s}) \\
0.91(\mathrm{~s}) \\
2.50 / 3.29(\text { each } \mathrm{d}, \\
J=14.4)\end{array}$ & $\begin{array}{c}3.25(\text { sept }, J=6.8) \\
1.21(\mathrm{~d}, J=6.8) \\
1.18(\mathrm{~d}, J=6.8) \\
0.88(\mathrm{~s}) \\
0.91(\mathrm{~s}) \\
2.50 / 3.29(\text { each } \mathrm{d}, \\
J=14.4)\end{array}$ & $\begin{array}{c}3.27(\text { sept }, J=6.8) \\
1.17(\mathrm{~d}, J=6.8) \\
1.24(\mathrm{~d}, J=6.8) \\
0.81(\mathrm{~s}) \\
0.92(\mathrm{~s}) \\
2.48 / 2.77(\text { each } \mathrm{d}, \\
J=17.0)\end{array}$ & $1.30(\mathrm{~s})$ & $1.29(\mathrm{~s})$ \\
\hline \multicolumn{6}{|l|}{ Part B } \\
\hline $1^{\prime}$ & & & & $\begin{array}{c}1.37(\mathrm{~m}) \\
3.17(\mathrm{dt}, J=13.6 \\
3.6)\end{array}$ & $\begin{array}{c}2.02(\mathrm{ddd}, J=13.2, \\
11.2,2.8) \\
2.92(\mathrm{dt}, J=13.2, \\
8.8)\end{array}$ \\
\hline $2^{\prime}$ & $6.91(\mathrm{~d}, J=8.0)$ & $6.91(\mathrm{dd}, J=8.0,1.6)$ & $6.92(\mathrm{~d}, J=8.0)$ & $1.71(\mathrm{~m})$ & $\begin{array}{l}2.30(\mathrm{~m}) \\
2.73(\mathrm{~m})\end{array}$ \\
\hline $\begin{array}{l}3^{\prime} \\
4^{\prime}\end{array}$ & $6.80(\mathrm{~d}, J=8.0)$ & $6.81(\mathrm{~d}, J=8.0)$ & $6.82(\mathrm{~d}, J=8.0)$ & $3.27(\mathrm{dd}, J=9.0,6.8)$ & \\
\hline $\begin{array}{l}T^{\prime} \\
5^{\prime}\end{array}$ & & & & $1.24(\mathrm{~m})$ & $2.11(\mathrm{~m})$ \\
\hline $6^{\prime}$ & $6.94(\mathrm{~s})$ & $6.94(\mathrm{~s})$ & $6.94(\mathrm{~s})$ & $\begin{array}{l}1.55(\mathrm{~m}) \\
1.78(\mathrm{~m})\end{array}$ & $\begin{array}{l}1.68(\mathrm{~m}) \\
1.74(\mathrm{~m})\end{array}$ \\
\hline $\begin{array}{c}7^{\prime} \\
8^{\prime} \\
9^{\prime} \\
10^{\prime} \\
11^{\prime} \\
12^{\prime} \\
13^{\prime}\end{array}$ & $5.06(\mathrm{~s})$ & $5.05(\mathrm{~s})$ & $5.12(\mathrm{~m})$ & $2.78(\mathrm{~m})$ & $2.80(\mathrm{~m})$ \\
\hline $14^{\prime}$ & & & & $6.43(s)$ & $6.48(\mathrm{~s})$ \\
\hline $15^{\prime}$ & & & & $3.10($ sept,$J=6.8)$ & $3.14($ sept,$J=6.8)$ \\
\hline $16^{\prime}$ & & & & $1.14(\mathrm{~d}, J=6.8)$ & $1.16(\mathrm{~d}, J=6.8)$ \\
\hline $17^{\prime}$ & & & & $1.23(\mathrm{~d}, J=6.8)$ & $1.16(\mathrm{~d}, J=6.8)$ \\
\hline $18^{\prime}$ & & & & $1.04(\mathrm{~s})$ & $1.15(\mathrm{~s})$ \\
\hline $19^{\prime}$ & & & & $0.86(\mathrm{~s})$ & $1.10(\mathrm{~s})$ \\
\hline $20^{\prime}$ & & & & $1.28(\mathrm{~s})$ & $1.17(\mathrm{~s})$ \\
\hline $1^{\prime \prime}$ & & & & & \\
\hline $2^{\prime \prime}$ & $6.44(\mathrm{~d}, J=8.0)$ & $6.42(\mathrm{~d}, J=8.0)$ & $6.37(\mathrm{dd}, J=8.0,1.6)$ & & \\
\hline $3^{\prime \prime}$ & $6.73(\mathrm{~d}, J=8.0)$ & $6.73(\mathrm{~d}, J=8.0)$ & $6.74(\mathrm{~d}, J=8.0)$ & & \\
\hline $\begin{array}{l}4^{\prime \prime} \\
5^{\prime \prime}\end{array}$ & & & & & \\
\hline $6^{\prime \prime}$ & $6.42(\mathrm{~s})$ & $6.41(\mathrm{~s})$ & $6.33(\mathrm{~s})$ & & \\
\hline $7^{\prime \prime}$ & $6.47(\mathrm{~s})$ & $6.44(\mathrm{~s})$ & 6.35 (br. s) & & \\
\hline $9^{\prime \prime}$ & $\begin{array}{c}3.94 / 4.81 \text { (each dd } \\
J=13.2,2.8)\end{array}$ & $\begin{array}{c}3.90(\mathrm{dd}, J=13.2, \\
2.8), \\
4.77(\mathrm{dd}, J=13.2 \\
1.6)\end{array}$ & $\begin{array}{l}3.90(\mathrm{dd}, J=13.6,2.4) \\
4.74(\mathrm{dd}, J=13.6,2.4)\end{array}$ & & \\
\hline $4^{\prime}, 5^{\prime}-\mathrm{OCH}_{2} \mathrm{O}-$ & $5.94(\mathrm{~s})$ & $5.94(\mathrm{~s})$ & $5.94(\mathrm{~s})$ & & \\
\hline $4^{\prime \prime}, 5^{\prime \prime}-\mathrm{OCH}_{2} \mathrm{O}-$ & $\begin{array}{l}5.97(\mathrm{~d}, J=1.6) \\
5.99(\mathrm{~d}, J=1.6)\end{array}$ & $\begin{array}{l}5.98(\mathrm{~d}, J=1.6) \\
6.01(\mathrm{~d}, J=1.6)\end{array}$ & $\begin{array}{l}5.98(\mathrm{~d}, J=1.6) \\
6.00(\mathrm{~d}, J=1.6)\end{array}$ & & \\
\hline
\end{tabular}


Table 2. ${ }^{13} \mathrm{C}$ NMR data for compounds $\mathbf{1}-\mathbf{5}\left(\delta\right.$ in ppm, $125 \mathrm{MHz}$ for $\left.{ }^{13} \mathrm{C} \mathrm{NMR} \mathrm{in} \mathrm{CDCl}_{3}\right)$.

\begin{tabular}{|c|c|c|c|c|c|}
\hline No & 1 & 2 & 3 & 4 & 5 \\
\hline \multicolumn{6}{|l|}{ A } \\
\hline 1 & 42.1 & 42.2 & 30.3 & 133.2 & 75.4 \\
\hline 2 & 18.8 & 18.7 & 16.0 & 24.3 & 24.6 \\
\hline 3 & 42.4 & 42.5 & 31.6 & 29.8 & 34.4 \\
\hline 4 & 34.3 & 34.4 & 31.8 & 71.2 & 69.5 \\
\hline 5 & 58.1 & 58.2 & 50.7 & 73.5 & 133.3 \\
\hline 6 & 23.8 & 23.8 & 40.0 & 134.9 & 137.7 \\
\hline 7 & 36.0 & 36.2 & 75.9 & 42.0 & 42.1 \\
\hline 8 & 122.1 & 122.1 & 134.7 & 22.3 & 21.5 \\
\hline 9 & 137.2 & 138.5 & 118.9 & 30.8 & 32.5 \\
\hline 10 & 70.9 & 71.2 & 80.1 & 144.6 & 75.8 \\
\hline 11 & 141.8 & 140.5 & 140.6 & 21.0 & 26.1 \\
\hline 12 & 137.3 & 138.0 & 137.6 & 20.1 & 17.0 \\
\hline 13 & 134.7 & 134.7 & 134.0 & 17.0 & 22.2 \\
\hline 14 & 119.0 & 118.2 & 114.6 & 108.4 & 23.0 \\
\hline 15 & 26.9 & 27.0 & 26.8 & 22.6 & 16.8 \\
\hline 16 & 22.7 & 22.8 & 23.1 & & \\
\hline 17 & 22.1 & 22.3 & 21.7 & & \\
\hline 18 & 21.6 & 21.7 & 26.9 & & \\
\hline 19 & 32.2 & 32.2 & 30.4 & & \\
\hline 20 & 40.6 & 40.5 & 38.8 & & \\
\hline \multicolumn{6}{|l|}{ Part B } \\
\hline $1^{\prime}$ & 127.2 & 127.1 & 126.9 & 35.6 & 37.1 \\
\hline $2^{\prime}$ & 107.6 & 108.4 & 107.5 & 28.3 & 34.2 \\
\hline $3^{\prime}$ & 108.5 & 108.5 & 108.4 & 78.7 & 220.0 \\
\hline $4^{\prime}$ & 148.0 & 147.9 & 147.7 & 39.3 & 47.2 \\
\hline $5^{\prime}$ & 148.3 & 148.3 & 148.37 & 52.5 & 51.8 \\
\hline $6^{\prime}$ & 121.1 & 121.40 & 121.16 & 19.1 & 20.7 \\
\hline $7^{\prime}$ & 77.4 & 76.92 & 76.86 & 32.5 & 31.9 \\
\hline $8^{\prime}$ & 78.6 & 79.08 & 78.78 & 127.5 & 127.6 \\
\hline $9^{\prime}$ & 171.7 & 171.50 & 171.39 & 123.4 & 135.0 \\
\hline $10^{\prime}$ & & & & 39.0 & 37.9 \\
\hline $11^{\prime}$ & & & & 139.8 & 138.4 \\
\hline $12^{\prime}$ & & & & 137.4 & 136.9 \\
\hline $13^{\prime}$ & & & & 134.2 & 132.3 \\
\hline $14^{\prime}$ & & & & 118.3 & 118.1 \\
\hline $15^{\prime}$ & & & & 26.8 & 26.8 \\
\hline $16^{\prime}$ & & & & 22.2 & 22.2 \\
\hline $17^{\prime}$ & & & & 22.4 & 22.4 \\
\hline $18^{\prime}$ & & & & 28.6 & 28.8 \\
\hline $19^{\prime}$ & & & & 15.9 & 19.7 \\
\hline $20^{\prime}$ & & & & 20.1 & 21.8 \\
\hline $1^{\prime \prime}$ & 129.6 & 129.6 & 129.9 & & \\
\hline $2^{\prime \prime}$ & 108.4 & 108.4 & 108.3 & & \\
\hline $3^{\prime \prime}$ & 108.5 & 108.5 & 108.4 & & \\
\hline $4^{\prime \prime}$ & 147.9 & 147.9 & 147.9 & & \\
\hline $5^{\prime \prime}$ & 147.7 & 147.9 & 147.9 & & \\
\hline $6^{\prime \prime}$ & 123.2 & 123.1 & 123.2 & & \\
\hline $7^{\prime \prime}$ & 128.7 & 128.6 & 128.4 & & \\
\hline $8^{\prime \prime}$ & 129.1 & 129.1 & 128.8 & & \\
\hline $9^{\prime \prime}$ & 69.6 & 69.6 & 69.6 & & \\
\hline $4^{\prime}, 5^{\prime}-\mathrm{OCH}_{2} \mathrm{O}-$ & 101.4 & 101.4 & 101.4 & & \\
\hline $4^{\prime \prime}, 5^{\prime \prime}-\mathrm{OCH}_{2} \mathrm{O}-$ & 101.4 & 101.4 & 101.4 & & \\
\hline
\end{tabular}

Compounds 1 and 2 are speculated to be $9(10 \rightarrow 20)$ abeoabietane type diterpenoids (demethylsalvicanol) combined with 8, $8^{\prime}$-lignans (Taiwanin A). After calculating the degree of unsaturation, subtracting the above inferred part, there is still 1 degree of unsaturation. Therefore, the combination of the two monomer compounds should be adjacent to the oxygen atom and cyclized. The Z-configuration of the extra-cyclic olefinic proton at C- $7^{\prime \prime}$ was confirmed by the observation of the ${ }^{1} \mathrm{H}-{ }^{1} \mathrm{H}$ NOESY correlation between $\mathrm{H}-7^{\prime \prime}$ and $\mathrm{H}-9^{\prime \prime}$ in compounds 1-3 (Table 3). 
Table 3. 2D-NMR data for compounds $\mathbf{1}-\mathbf{5}$ in $\mathrm{CDCl}_{3}$ ( $\delta$ in ppm, $J$ in $\mathrm{Hz}, 500 \mathrm{MHz}$ in $\mathrm{CDCl}_{3}$ ).




Table 3. Cont.

\begin{tabular}{|c|c|c|c|c|c|c|c|c|c|c|c|c|c|c|c|}
\hline No & & 1 & & & 2 & & & 3 & & 4 & & & 5 & & \\
\hline \multicolumn{16}{|l|}{ Part B } \\
\hline $1^{\prime}$ & & & & & & & & & & $2^{\prime}$ & $2^{\prime}$ & & $2^{\prime}$ & $2^{\prime}$ & $20^{\prime}$ \\
\hline $2^{\prime}$ & & & & & & & & & & $1^{\prime}$ & & $\begin{array}{l}1^{\prime}, 3^{\prime}, 19^{\prime}, \\
20^{\prime}\end{array}$ & $1^{\prime}$ & $3^{\prime}, 10^{\prime}$ & \\
\hline $3^{\prime}$ & & & & & & & & & & $2^{\prime}$ & $2^{\prime}, 4^{\prime}$ & & & & \\
\hline $\begin{array}{l}4^{\prime} \\
5^{\prime}\end{array}$ & $6^{\prime}$ & & & $6^{\prime}$ & & & $6^{\prime}$ & & & $6^{\prime}$ & $3^{\prime} \quad 7^{\prime} \quad 10^{\prime}$ & & & & $6^{\prime}$ \\
\hline $6^{\prime}$ & $5^{\prime}$ & $7^{\prime}$ & & $\begin{array}{l}6 \\
5^{\prime}\end{array}$ & $7^{\prime}$ & & $\begin{array}{l}6^{\prime} \\
5^{\prime}\end{array}$ & $7^{\prime}$ & & $\begin{array}{c}6^{\prime} \\
5^{\prime}, 7^{\prime}\end{array}$ & $3,7,10^{\prime}$ & $\begin{array}{c}3^{\prime}, 6^{\prime} \\
5^{\prime}, 7^{\prime}, 19^{\prime}\end{array}$ & $\begin{array}{c}6^{\prime} \\
5^{\prime}, 7^{\prime}\end{array}$ & $7^{\prime}, 8^{\prime}, 10^{\prime}$ & $6^{\prime}$ \\
\hline $7^{\prime}$ & & $2^{\prime}, 8^{\prime}, 8^{\prime \prime}$ & $2^{\prime}$ & & $2^{\prime}, 8^{\prime}{ }^{\prime \prime}, 12^{\prime}$, & $2^{\prime}$ & & $\begin{array}{c}2^{\prime}, 8^{\prime}, 12^{\prime}, \\
8^{\prime \prime}\end{array}$ & $2^{\prime}$ & & $\begin{array}{c}5^{\prime}, 6^{\prime}, 8^{\prime} \\
14^{\prime}\end{array}$ & $6^{\prime}, 14^{\prime}$ & & $6^{\prime}, 9^{\prime}$ & \\
\hline $\begin{array}{c}8^{\prime} \\
9^{\prime} \\
10^{\prime} \\
11^{\prime} \\
12^{\prime} \\
13^{\prime}\end{array}$ & & & & & & & & & & & & & & & \\
\hline $14^{\prime}$ & & & & & & & & & & & $\begin{array}{c}9^{\prime}, 12^{\prime}, \\
15^{\prime}\end{array}$ & $7^{\prime}, 17^{\prime}$ & & $\begin{array}{c}7^{\prime}, 12^{\prime} \\
13^{\prime}, 15^{\prime}\end{array}$ & $17^{\prime}$ \\
\hline $15^{\prime}$ & & & & & & & & & & $16^{\prime}, 17^{\prime}$ & $16^{\prime}, 17^{\prime}$ & & $16^{\prime}, 17^{\prime}$ & & \\
\hline $16^{\prime}$ & & & & & & & & & & $15^{\prime}$ & $13^{\prime}$ & & $15^{\prime}$ & & \\
\hline $18^{\prime}$ & & & & & & & & & & & $3^{\prime}, 5^{\prime}$ & $3^{\prime}$ & & $\begin{array}{c}3^{\prime}, 4^{\prime}, 5^{\prime}, \\
19^{\prime}\end{array}$ & $5^{\prime}$ \\
\hline $19^{\prime}$ & & & & & & & & & & & $3^{\prime}, 5^{\prime}$ & $2^{\prime}, 6^{\prime}, 20^{\prime}$ & & $3^{\prime}, 4^{\prime}, 18^{\prime}$ & $6^{\prime}$ \\
\hline $20^{\prime}$ & & & & & & & & & & & $1^{\prime}, 5^{\prime}, 10^{\prime}$ & $2^{\prime}, 19^{\prime}$ & & $5^{\prime}, 9^{\prime}$ & \\
\hline \multicolumn{16}{|l|}{$1^{\prime \prime}$} \\
\hline \multicolumn{16}{|l|}{$2^{\prime \prime}$} \\
\hline \multicolumn{16}{|l|}{$\begin{array}{l}3^{\prime \prime} \\
4^{\prime \prime}\end{array}$} \\
\hline $5^{\prime \prime}$ & $6^{\prime \prime}$ & & & $6^{\prime \prime}$ & & & $6^{\prime \prime}$ & & & & & & & & \\
\hline $6^{\prime \prime}$ & $5^{\prime \prime}$ & & & $5^{\prime \prime}$ & & & $5^{\prime \prime}$ & & & & & & & & \\
\hline $7^{\prime \prime}$ & & $\begin{array}{l}2^{\prime \prime}, 6^{\prime \prime} \\
8^{\prime \prime}\end{array}$ & $9^{\prime \prime}$ & & $\begin{array}{l}2^{\prime \prime}, 6^{\prime \prime} \\
8^{\prime \prime}\end{array}$ & $9^{\prime \prime}$ & & $\begin{array}{c}2^{\prime \prime}, 6^{\prime \prime} \\
8^{\prime \prime}\end{array}$ & $9^{\prime \prime}$ & & & & & & \\
\hline $\begin{array}{l}8^{\prime} ; \\
9^{\prime \prime}\end{array}$ & & & $x^{\prime}$ & & & & & & & & & & & & \\
\hline $4^{\prime}, 5^{\prime}-$ & & & $2^{\prime}$ & & & $2^{\prime}$ & & & $2^{\prime}$ & & & & & & \\
\hline $\begin{array}{c}\mathrm{OCH}_{2} \mathrm{O}- \\
4^{\prime \prime}, 5^{\prime \prime}-\end{array}$ & & & & & & & & & & & & & & & \\
\hline $\mathrm{OCH}_{2} \mathrm{O}-$ & & & & & & & & & & & & & & & \\
\hline
\end{tabular}


Compounds $\mathbf{1}$ and $\mathbf{2}$ are pure compounds obtained from different fractions, and their NMR spectra are very similar. The main difference between the two is that the optical rotation values are, respectively, positive $\left([\alpha]_{D}^{30}=+131.7\right)$ and negative $\left([\alpha]_{D}^{30}=\right.$ -26.2). Although there are other chiral centers, the absolute configurations of Parts $A$ and $\mathrm{B}$ have been confirmed according to the literature. Therefore, it can only be based on the large difference between the positive and negative values of the optical rotation value that the relative stereo configuration of the $C-7^{\prime}$ position should be the opposite. Compounds 1 and 2 were a pair of $C-7^{\prime}$ stereoisomerism. Therefore, we temporarily decided that the relative configuration at $C-7^{\prime}$ of $\mathbf{1}$ is $\beta$-orientation. According to the reverse optical rotation value, the relative configuration at C-7 of 2 is proposed as $\alpha$-form. However, the absolute configurations of Compounds $\mathbf{1}$ and $\mathbf{2}$ in C-7' and C- $8^{\prime}$ are uncertain. These data, supported by the ${ }^{13} \mathrm{C}$ NMR (Table 2), DEPT, COSY (Table 3), HSQC, and HMBC (Table 3) spectra, were in agreement with a dimer system bearing two partial structures $\mathbf{A}$ and $\mathbf{B}$. The structures of $\mathbf{1}$ and $\mathbf{2}$ were established as taiwaniacryptodimers $\mathrm{A}$ and B (rel-(3S,12aS,Z)-3-(benzo[d][1,3]dioxol-5-yl)-4'-(benzo[d][1,3]dioxol-5-ylmethylene)12a-hydroxy-5-isopropyl-9,9-dimethyl-4' ${ }^{\prime} 5^{\prime}, 8,8 \mathrm{a}, 9,10,11,12,12 \mathrm{a}, 13$-decahydro-2' $\mathrm{H}, 3 \mathrm{H}, 7 \mathrm{H}$ spiro [benzo[ $\left[5^{\prime}, 6^{\prime}\right]$ cyclohepta[1 $\left.1^{\prime}, 2^{\prime}: 3,4\right]$ benzo[1,2-b][1,4]dioxine-2,3'-furan]-2' -one and rel(3R,12aS,Z)-3-(benzo[d][1,3]dioxol-5-yl)-4'-(benzo[d][1,3]dioxol-5-ylmethylene)-12a-hydr oxy-5-isopropyl-9,9-dimethyl-4 ${ }^{\prime}, 5^{\prime}, 8,8 \mathrm{a}, 9,10,11,12,12 \mathrm{a}, 13$-decahydro-2' $\mathrm{H}, 3 \mathrm{H}, 7 \mathrm{H}$-spiro[benz o[5', $\left.6^{\prime}\right]$ cyclohepta $\left[1^{\prime}, 2^{\prime}: 3,4\right]$ benzo[1,2- $\left.b\right][1,4]$ dioxine-2, $3^{\prime}$-furan $]-2^{\prime}$-one), respectively.

Compound 3 was obtained as an optically active colorless gum. $[\alpha]_{\mathrm{D}}^{30}=+105.4(c=0.6$, $\left.\mathrm{CHCl}_{3}\right)$. The molecular formula was determined as $\mathrm{C}_{40} \mathrm{H}_{40} \mathrm{O}_{9}$ based on the $[\mathrm{M}]^{+}$peak at $m / z 664.2668$ (calcd. 664.2673 for $\left.\mathrm{C}_{40} \mathrm{H}_{40} \mathrm{O}_{9}\right)$ in its HR-EI-MS. UV absorptions $\left(\lambda_{\max }\right.$ bands at 227, 284, and $308 \mathrm{~nm}$ ) confirmed the presence of a benzenoid nucleus. The IR spectrum revealed the presence of ester lactone $\left(1791 \mathrm{~cm}^{-1}\right)$, and aromatic rings $(1618$, 1498 , and $\left.1447 \mathrm{~cm}^{-1}\right)$, respectively. Twenty-one degrees of insaturation were determined from the molecular formula, ${ }^{13} \mathrm{C}-\mathrm{NMR}$ (Table 1), and DEPT spectra. Because part of the all spectra data of $\mathbf{3}$ were similar to $\mathbf{1}$ and $\mathbf{2}$, it is inferred from the molecular formula and mass fragments that Compound 3 is also a dimer compound. From the ${ }^{1} \mathrm{H}-\mathrm{NMR}$ spectrum, there are isopropyl groups attached to the benzene ring $\left(\delta_{\mathrm{H}} 1.24(\mathrm{~d}, J=6.8 \mathrm{~Hz}, \mathrm{H}-17), 1.17\right.$ $(\mathrm{d}, J=6.8 \mathrm{~Hz}, \mathrm{H}-16), 3.27$ (sept, $J=6.8 \mathrm{~Hz}, \mathrm{H}-15)$, two singlet methyl groups $\left(\delta_{\mathrm{H}} 0.81\right.$ (s, $\mathrm{H}-18$ ), and 0.92 (s, H-19)).

The splitting of two groups of tri-substituted benzene ring $\mathrm{ABX}$ patterns $\left[\delta_{\mathrm{H}} 6.82(1 \mathrm{H}\right.$, $\left.\mathrm{d}, J=8.0 \mathrm{~Hz}, \mathrm{H}-3^{\prime}\right), 6.92\left(1 \mathrm{H}, \mathrm{d}, J=8.0 \mathrm{~Hz}, \mathrm{H}-2^{\prime}\right), 6.94\left(1 \mathrm{H}, \mathrm{s}, \mathrm{H}-6^{\prime}\right)$, and $\delta_{\mathrm{H}} 6.33\left(1 \mathrm{H}, \mathrm{s}, \mathrm{H}-6^{\prime \prime}\right)$, $\left.6.37\left(1 \mathrm{H}, \mathrm{dd}, J=8.0,1.6 \mathrm{~Hz}, \mathrm{H}-2^{\prime \prime}\right), 6.74\left(1 \mathrm{H}, \mathrm{d}, J=8.0 \mathrm{~Hz}, \mathrm{H}-3^{\prime \prime}\right)\right]$, two $-\mathrm{OCH}_{2} \mathrm{O}-$ signals [ $\delta_{\mathrm{H}} 5.94\left(2 \mathrm{H}, \mathrm{s}, \mathrm{C}^{\prime}, 5^{\prime}-\mathrm{OCH}_{2} \mathrm{O}-\right.$ ) and 5.98/6.00 (each $\left.1 \mathrm{H}, \mathrm{J}=1.6 \mathrm{~Hz}, \mathrm{C}^{\prime \prime}, 5^{\prime \prime}-\mathrm{OCH}_{2} \mathrm{O}-\right)$ ]. In conjunction with the ${ }^{13} \mathrm{C}-\mathrm{NMR}$ spectrum, there is a lactone signal at $\delta_{\mathrm{C}} 171.4\left(\mathrm{C}-9^{\prime}\right)$. From the above characteristics, we know that Compound $\mathbf{3}$ is similar to $\mathbf{1}$ and $\mathbf{2}$. The main difference is that the part A C-7 of 3 changes from a secondary carbon to an oxygenated tertiary carbon, which is a $9(10 \rightarrow 20)$ abeoabietane diterpenoids combined with $8,8^{\prime}$-lignan. It can also be known from the IR spectrum that there is no absorption of hydroxyl groups. The C-7 and C-10 of the Part A form epoxide through the connection of oxygen atoms, which is also consistent with the calculated degree of unsaturation. Part A can be confirmed by comparison with the known compound brussonol (5,6-dihydroxysalviasperanol) [17,18].

The structure was further confirmed by ${ }^{13} \mathrm{C}$ NMR (Table 1), DEPT, COSY (Table 3), and NOESY (Table 3) experiments. Thus, the structure of 3 was determined to be $\left(3^{\prime} S^{*}, 7^{\prime} S^{*}, 8 \mathrm{a}^{\prime} S^{*}\right.$, $\left.12 \mathrm{a}^{\prime} S^{*}, Z\right)-3^{\prime}$-(benzo[d][1,3]dioxol-5-yl)-4-(benzo[d][1,3]dioxol-5-ylmethylene)-5'-isopropyl$9^{\prime}, 9^{\prime}$-dimethyl-4,5,7', $8^{\prime}, 8 \mathrm{a}^{\prime}, 9^{\prime}, 10^{\prime}, 11^{\prime}, 12^{\prime}, 13^{\prime}$-decahydro- $2 H, 3^{\prime} H$-spiro[furan-3,2' -[7,12a]epoxy benzo[ $\left[5^{\prime}, 6^{\prime}\right]$ cyclohepta[ $\left[1^{\prime}, 2^{\prime}: 3,4\right]$ benzo[1,2- $\left.b\right][1,4]$ dioxin]-2-one and was named Taiwaniacryptodimer $\mathrm{C}$.

Compound 4 was isolated as a yellowish gum; its molecular formula $\mathrm{C}_{35} \mathrm{H}_{50} \mathrm{O}_{3}$ was established by ${ }^{13} \mathrm{C}-\mathrm{NMR}$ and HR-EI-MS data, and the eleven degrees of insaturation were determined from the molecular formula, the ${ }^{13} \mathrm{C}$-NMR spectrum, and the DEPT experiment (Table 1). Analysis of its IR spectrum suggested that 4 contains an $\mathrm{OH}$ group $\left(3423 \mathrm{~cm}^{-1}\right)$ 
and an aromatic moiety $\left(1615,1464 \mathrm{~cm}^{-1}\right)$. The ${ }^{13} \mathrm{C}$ - and ${ }^{1} \mathrm{H}-\mathrm{NMR}$ spectrum (Table 1$)$ data together with the UV absorption bands at $\lambda_{\max } 214$ and $234 \mathrm{~nm}$ suggested that Compound 4 is very similar to hinokione [19].

The ${ }^{1} \mathrm{H}$ NMR spectrum shows that there are two isopropyl groups $\left[\delta_{\mathrm{H}} 1.14(\mathrm{~d}\right.$, $\left.\left.J=6.8 \mathrm{~Hz}, \mathrm{H}-16^{\prime}\right), 1.23\left(\mathrm{~d}, J=6.8 \mathrm{~Hz}, \mathrm{H}-17^{\prime}\right)\right), 3.10\left(\mathrm{sept}, J=6.8 \mathrm{~Hz}, \mathrm{H}-15^{\prime}\right)$ ) and $\delta_{\mathrm{H}}$ $0.75((\mathrm{~d}, J=6.8 \mathrm{~Hz}, \mathrm{H}-13), 0.94(\mathrm{~d}, J=6.8 \mathrm{~Hz}, \mathrm{H}-12), 2.28(\mathrm{~m}, \mathrm{H}-11)]$, and one pentasubstituted benzene proton at $\delta_{\mathrm{H}} 6.43\left(\mathrm{~s}, \mathrm{H}-14^{\prime}\right)$, one terminal double bond at $\delta_{\mathrm{H}} 4.77 / 4.93$ (each $\left.\mathrm{s}, \mathrm{CH}_{2}-14\right)$, 4 singlet methyls at $\delta_{\mathrm{H}} 0.86\left(\mathrm{~s}, \mathrm{CH}_{3}-19^{\prime}\right), 1.04\left(\mathrm{~s}, \mathrm{CH}_{3}-18^{\prime}\right), 1.28\left(\mathrm{~s}, \mathrm{CH}_{3}-20^{\prime}\right)$, and $1.30\left(\mathrm{~s}, \mathrm{CH}_{3}-15\right)$. The ${ }^{13} \mathrm{C}$ NMR spectrum shows that 10 olefinic carbons are forming a benzene ring, a terminal double bond, a four-substituted double bond, and three oxygenated carbons at $\delta_{C} 78.7\left(\mathrm{C}-3^{\prime}\right), 73.5(\mathrm{C}-5), 71.2(\mathrm{C}-4)$. From the above signals, it is inferred that it is a dimer formed by combining two monomers (A and B). Part A is a cadinane and Part B is an abietane skeleton.

Part B can be compared with the known compound hinokione: (1) C-3' changes from a carbonyl group to a hydroxyl group, so the ${ }^{13} \mathrm{C}$ NMR spectrum also shifts from $\delta_{C} 220.3$ to $78.7\left(C-3^{\prime}\right)$. (2) Except for $C-2^{\prime}, 3^{\prime}, 4^{\prime}, 11^{\prime}, 12^{\prime}$, which are affected by different functional groups, the ${ }^{1} \mathrm{H}$ NMR and ${ }^{13} \mathrm{C}$ NMR data have high similarity. After calculating the degree of unsaturation, subtracting the four unsaturations in Part $\mathrm{A}$ and six unsaturations in Part B, there is one unsaturation left. Therefore, the combination of two monomer compounds should be an adjacent dimer to the oxygen atom and cyclized. Based on further spectral data, the structure of 4 was established to be taiwaniacryptodimer $\mathrm{D}$ (rel-(3S,4aR,9aR,15S,15bS,16cS)-8,15-diisopropyl-4,4,9a,16c-tetramethyl-12-methylene$1,2,3,4,4 a, 5,6,9 a, 10,11,12,13,14,15,15 b, 16 c-h e x a d e c a h y d r o n a p h t h o[1,2-b]$ phenanthro $[3,4-$ e][1,4]dioxin-3-ol).

Compound 5 was obtained as yellowish gum; it has the formula $\mathrm{C}_{35} \mathrm{H}_{50} \mathrm{O}_{4}$ according to the HR-EI-MS and ${ }^{13} \mathrm{C}-\mathrm{NMR}$ data. It has an IHD of 11 as deduced from its molecular formula. The IR spectrum shows absorptions for an $\mathrm{OH}$ group $\left(3453 \mathrm{~cm}^{-1}\right)$, a carbonyl group (17121 cm_1), and an aromatic moiety (1464 and $\left.1421 \mathrm{~cm}^{-1}\right)$. The UV spectra of 5 confirmed the presence of an aromatic group $\left(\lambda_{\max } 231\right.$ and $\left.286 \mathrm{~nm}\right)$. The ${ }^{1} \mathrm{H} \mathrm{NMR}$ spectrum of 5 (Table 1) displayed resonances for two iPr moieties $\left[\delta_{\mathrm{H}} 0.84(\mathrm{~d}, J=6.8 \mathrm{~Hz}\right.$, $\mathrm{H}-12), 0.94(\mathrm{~d}, J=6.8 \mathrm{~Hz}, \mathrm{H}-13), 2.12(\mathrm{~m}, \mathrm{H}-11)$ and $\delta_{\mathrm{H}} 1.16 / 1.16$ (each $3 \mathrm{H}, \mathrm{d}, J=6.8 \mathrm{~Hz}, \mathrm{H}-$ $\left.16^{\prime} / 17^{\prime}\right), 3.14$ (sept, $\left.\left.J=6.8 \mathrm{~Hz}, \mathrm{H}-15^{\prime}\right)\right]$, a trisubstituted double bond $\left[\delta_{\mathrm{H}} 5.65(\mathrm{~d}(\mathrm{~d}, J=2.0 \mathrm{~Hz}\right.$, $\mathrm{H}-5)]$, a five-substituted benzene ring $\left[\delta_{\mathrm{H}} 6.48\left(\mathrm{~s}, \mathrm{H}-14^{\prime}\right)\right]$, and five singlet methyl groups [ $\delta_{\mathrm{H}} 1.29\left(\mathrm{~s}, \mathrm{CH}_{3}-15\right), 1.30\left(\mathrm{~s}, \mathrm{CH}_{3}-14\right), 1.10\left(\mathrm{~s}, \mathrm{CH}_{3}-19^{\prime}\right), 1.15\left(\mathrm{~s}, \mathrm{CH}_{3}-18^{\prime}\right), 1.17\left(\mathrm{~s}, \mathrm{CH}_{3}-\right.$ $\left.20^{\prime}\right)$. The ${ }^{13} \mathrm{C}-\mathrm{NMR}$ and DEPT experiments revealed that there is a carbonyl signal of $\delta_{\mathrm{C}}$ $220.0\left(\mathrm{C}-3^{\prime}\right), 8$ olefinic carbons form a benzene ring and a trisubstituted double bond, and three oxygen-containing carbons at $\delta_{C} 69.5(\mathrm{C}-4), 75.4(\mathrm{C}-1), 75.8(\mathrm{C}-10)$. From the above signals, it is inferred that it is a dimer compound formed by combining two monomers. Part A is a cadinane, and B is an abietane compound. Part B can be compared with the known compound hinokione: except for C-1, 11, and 12, which are affected by different functional groups, the ${ }^{1} \mathrm{H}$ NMR and ${ }^{13} \mathrm{C}$ NMR spectra are very nearly the same. After calculating the degree of unsaturation, subtracting the seven unsaturations in Part $B$ and three unsaturations in Part A, there is only one unsaturation left. Therefore, combining the two monomer compounds should be adjacent to the oxygen atom to cyclize to Compound 5. Finally, the 2D-NMR results are shown in Table 3. Consequently, Compound 5 was named taiwaniacryptodimer E (rel-(4aR,9aS,12R,14S,16aR,17cS)-12-hydroxy-8,14diisopropyl-4,4,12,16a,17c-pentamethyl-1,4,4a,5,6,11,12,14,15,16,16a,17c-dodecahydro-10Hnaphtho[1,8a- $b]$ phenanthro[3,4-e][1,4]dioxin-3(2H)-one).

\subsection{Biological Studies}

The antifungal activities of the roots of Taiwania cryptomerioides were tested against the following fungi: Aspergillus niger (BCRC-31512), Penicillium italicum (BCRC-30567), Candida albicans (BCRC-21538), and Saccharomyces cerevisiae (BCRC-20822). The antifungal data are 
shown in Table 4 and clinically used antifungal agent, ketoconazole, was used as positive control.

Table 4. Antifungal activity of five sufficient compounds isolated from the roots of Taiwania cryptomerioides (diameter of the zone of growth inhibition fungicidal zone in $\mathrm{mm}$, including the diameter of the disc, $8 \mathrm{~mm})$.

\begin{tabular}{ccccccc}
\hline $\begin{array}{c}\text { Test } \\
\text { Microorganism }\end{array}$ & \multicolumn{7}{c}{ Isolated Compounds } \\
\hline & $\mathbf{1}$ & $\mathbf{2}$ & $\mathbf{3}$ & $\mathbf{4}$ & $\mathbf{5}$ & Ketoconazole \\
\hline A. niger & $25.2 \pm 0.2$ & $20.2 \pm 1.9$ & $12 \pm 2.9$ & $20 \pm 1.8$ & $22.5 \pm 2.2$ & $32 \pm 1.2$ \\
P. italicum & $20.0 \pm 0.2$ & $18.2 \pm 1.6$ & $11.4 \pm 2.2$ & $26.3 \pm 1.1$ & $12.3 \pm 0.3$ & $30 \pm 1.4$ \\
C. albicans & $22.2 \pm 0.2$ & $17.0 \pm 0.2$ & - & $19 \pm 2.2$ & $18.0 \pm 1.3$ & $30 \pm 5.4$ \\
S. cerevisiae & $21.2 \pm 1.8$ & $25.1 \pm 1.2$ & - & $20 \pm 1.3$ & $24.9 \pm 1.4$ & $33 \pm 1.8$ \\
\hline
\end{tabular}

Inhibition zone diameter (mm); - = no Inhibition zone; Positive control (STD): ketoconazole, Each value represents the mean \pm SD.

Our results indicated that Metabolites 1, 2, 4, and 5 present moderate antifungal activities compared with ketoconazole, for which Compound $\mathbf{3}$ was weak. From the results of the antifungal tests, the following conclusions can be drawn regarding these isolates: (a) Among the novel, Dimers 1 and 2 showed antifungal activities with inhibition zones of 25, 20, 22, and 21; 20, 18, 17, and $25 \mathrm{~mm}$ against Aspergillus niger (BCRC-31512) Penicillium italicum (BCRC-30567), Candida albicans (BCRC-21538), and Saccharomyces cerevisiae (BCRC20822), respectively. (b) The epoxide Dimer 3, taiwaniacryptodimer C (3), exhibited weak antifungal activities against Aspergillus niger (BCRC-31512) and Penicillium italicum (BCRC30567) strains. (c) The other type Dimers 4 and 5, taiwaniacryptodimer D and E (4 and 5) indicated the effective inhibition zones of 20, 26, 19, and 20;22, 12, 18, and $25 \mathrm{~mm}$ against Aspergillus niger (BCRC-31512) Penicillium italicum (BCRC-30567), Candida albicans (BCRC-21538), and Saccharomyces cerevisiae (BCRC-20822), respectively.

Compounds 1, 2, 4, and 5 were further tested for their inhibitory activity against A. niger, P. italicum, C. albicans, and S. cerevisiae by using a method as described in the experimental section (Table 5). Compound 1 was found to show inhibitory activity against A. niger strain with MIC value $54.87 \mu \mathrm{g} / \mathrm{mL}$. Compounds 2 and 4 were found to show inhibitory activity against $S$. cerevisiae or P. italicum strain with MIC values 58.92 and $42.78 \mu \mathrm{g} / \mathrm{mL}$. Compound 5 was also revealed to show inhibitory activity against $A$. niger and S. cerevisiae with MIC values of 62.86 and $56.32 \mu \mathrm{g} / \mathrm{mL}$, respectively. Their bioactivity was weaker than reference compound ketoconazole (with MIC values of 3.25, 6.72, 11.79, and $3.16 \mu \mathrm{g} / \mathrm{mL}$ for A. niger, P. italicum, C. albicans, and S. cerevisiae, respectively). No antifungal activity (MIC > 100) was observed for Compound 3 at concentrations below $100 \mu \mathrm{g} / \mathrm{mL}$ in this bioassay.

Table 5. MIC values of compounds $\mathbf{1}-\mathbf{5} \mathrm{in} \mu \mathrm{g} / \mathrm{mL}$ against four fungi strains.

\begin{tabular}{ccccc}
\hline Compounds & A. niger & P. italicum & C. albicans & S. cerevisiae \\
\hline $\mathbf{1}$ & $54.87 \pm 6.13^{\mathrm{a}}$ & $>100$ & $>100$ & $>100$ \\
$\mathbf{2}$ & $>100$ & $>100$ & $>100$ & $58.92 \pm 9.30^{\mathrm{a}}$ \\
$\mathbf{3}$ & $>100$ & $>100$ & $>100$ & $>100$ \\
$\mathbf{4}$ & $>100$ & $42.78 \pm 5.23^{\mathrm{a}}$ & $>100$ & $>100$ \\
$\mathbf{5}$ & $62.86 \pm 8.04^{\mathrm{a}}$ & $>100$ & $>100$ & $56.32 \pm 13.19^{\mathrm{a}}$ \\
Ketoconazole $^{\mathrm{a}}$ & $3.25 \pm 1.48^{\mathrm{a}}$ & $6.72 \pm 2.23^{\mathrm{a}}$ & $11.79 \pm 4.81^{\mathrm{a}}$ & $3.16 \pm 1.51^{\mathrm{a}}$ \\
\hline
\end{tabular}

a Each value represents the mean \pm SD.

Among $9(10 \rightarrow 20)$ abeoabietane type diterpenoids combined with $8,8^{\prime}$-lignans series analogues 1-3, taiwaniacryptodimers C (3) (possess the C-7 and C-10 of the part A form epoxide through the connection of oxygen atoms exhibited less effective inhibition than 
their analogues, taiwaniacryptodimers A (1) and B (2). Compounds 1 and 2 are a pair of diastereomers, with little difference in activity. Compounds $\mathbf{4}$ and $\mathbf{5}$ are dimers that belong to the combination of abietane and cadinane skeleton compounds. It has been reported in the literature that the compounds in the heartwood of T. cryptomerioides have good anti-fungal and anti-wood-destroying fungus effects, which shows that the extracted components of T. cryptomerioides have the potential to be used as food additives or health-care medical supplies, etc. As for how to develop and utilize, further research and exploration are needed.

\section{Materials and Methods}

\subsection{General Experimental Procedures}

TLC: silica gel $60 \mathrm{~F}_{254}$ precoated plates (Merck, Darmstadt, German). Column chromatography (CC): silica gel 60 (70-230 or 230-400 mesh, Merck) and Spherical C18 100A Reversed Phase Silica Gel (RP-18) (particle size: 20-40 $\mu \mathrm{m}$ ) (Silicycle, Quebec, Canada). HPLC: Spherical C18 column $(250 \times 10 \mathrm{~mm}, 5 \mu \mathrm{m})$ (Waters, Milford, MA, USA); LDCAnalytical-III apparatus. UV Spectra: Jasco UV-240 spectrophotometer; $\lambda_{\max }(\log \varepsilon)$ in $\mathrm{nm}$. Optical rotation: Jasco DIP-370 polarimeter; in $\mathrm{CHCl}_{3}$. IR Spectra: Perkin-Elmer-2000 FT-IR spectrophotometer; $v$ in $\mathrm{cm}^{-1} .{ }^{1} \mathrm{H}-,{ }^{13} \mathrm{C}$ - and $2 \mathrm{D}-\mathrm{NMR}$ spectra were obtained utilizing a Varian-Mercury-500 and Varian-Unity-Plus-400 instrument and reported in $\mathrm{CDCl}_{3} \cdot{ }^{1} \mathrm{H}$ and ${ }^{13} \mathrm{CNMR}$ chemical shifts are reported in ppm relative to either TMS $\left({ }^{1} \mathrm{H}\right)(\delta=0 \mathrm{ppm}$, J in $\mathrm{Hz})$ as an internal standard or the residual solvent peak as following: $\mathrm{CDCl}_{3}=7.26\left({ }^{1} \mathrm{H}\right.$ $\mathrm{NMR}), \mathrm{CDCl}_{3}=77.0\left({ }^{13} \mathrm{C}\right.$ NMR). ESI and HRESIMS: Bruker APEX-II mass spectrometer; in $m / z$.

\subsection{Plant Material}

The roots of T. cryptomerioides were collected from Taichung, Taiwan, in August 1996. The plant was identified by Dr. Shang-Tzen Chang, Professor of the Department of Forestry, National Taiwan University. A voucher specimen (no. 013542) has been deposited in the Herbarium of the Department of Botany of the National Taiwan University, Taipei, Taiwan.

\subsection{Isolation and Characterization of Secondary Metabolites}

Air-dried roots of T. cryptomerioides $(15 \mathrm{~kg})$ were extracted three times with $\mathrm{MeOH}(150$ L) at r.t. (7 days thrice). The methanol extract was concentrated, the brown residue suspended in $\mathrm{H}_{2} \mathrm{O}(7 \mathrm{~L})$ and then extracted with EtOAc, and the EtOAc fraction $(365 \mathrm{~g})$ subjected to CC (silica gel, hexane/EtOAc of increasing polarity $(\mathrm{H}(100) \rightarrow \mathrm{H}: \mathrm{E}(98: 2) \rightarrow \mathrm{H}: \mathrm{E}(97: 3) \rightarrow \mathrm{H}$ : $\mathrm{E}(95: 5) \rightarrow \mathrm{H}: \mathrm{E}(90: 10) \rightarrow \mathrm{H}: \mathrm{E}(80: 20) \rightarrow \mathrm{H}: \mathrm{E}(70: 30) \rightarrow \mathrm{H}: \mathrm{E}(60: 40) \rightarrow \mathrm{H}: \mathrm{E}(50: 50) \rightarrow \mathrm{H}: \mathrm{E}$ $(30: 70) \rightarrow \mathrm{E}(100)$; then using EtOAc/Acetone of increasing polarity $(\mathrm{E}(100) \rightarrow \mathrm{E}: \mathrm{A}(70: 30))$ to give 13 fractions (1-13), and each product fraction further purified by HPLC. Fraction 6 (4.4 g) was applied to a silica gel column (230-400 mesh, $40 \mathrm{~g})$, eluting with a gradient of nhexane-EtOAc, to obtain 11 fractions (6-1-6-11). Fractions 6-8 (41 mg) were applied by HPLC $\left(10 \%\right.$ EtOAc $\left./ \mathrm{CH}_{2} \mathrm{Cl}_{2}\right)$ to obtain Taiwaniacryptodimer E $(5 ; 4.7 \mathrm{mg})$. Fraction 7 (13.2 g) was chromatographed on a silica gel column (230-400 mesh, $650 \mathrm{~g}$ ), eluting with $\mathrm{CH}_{2} \mathrm{Cl}_{2}$-acetone (10:1), to give 4 fractions: Fr. 7.1 7.4. Fr. 7.1 (18.1 g) was purified by semi-preparative HPLC $\left(10 \% \mathrm{EtOAc} / \mathrm{CH}_{2} \mathrm{Cl}_{2}\right.$, flow rate $\left.2.5 \mathrm{~mL} / \mathrm{min}\right)$ to yield Taiwaniacryptodimers $\mathrm{A}-\mathrm{D}(\mathbf{1}-4 ; 1.0$, $1.0,7.2$, and $8.5 \mathrm{mg})$.

Taiwaniacryptodimer A (1): gum; $[\alpha]_{\mathrm{D}}^{30}=+131.7$ ( c 0.09, $\left.\mathrm{CHCl}_{3}\right)$; UV (MeOH): 229.0 (4.46), 284.0 (4.19), 314.0 (4.00) nm; IR (Neat): 3582 (-OH), 1790 (ester C=O), 1615, 1498 (benzene ring) $\mathrm{cm}^{-1} ;{ }^{1} \mathrm{H} \mathrm{NMR}\left(500 \mathrm{MHz}, \mathrm{CDCl}_{3}\right)$ : see Table $1 ;{ }^{13} \mathrm{C} \mathrm{NMR}\left(125 \mathrm{MHz}, \mathrm{CDCl}_{3}\right)$ : see Table 2); EIMS (70 eV) m/z (\%):666 ([M] $\left.]^{+}, 2\right), 368$ (8), 350 (64), 84 (100); HREIMS $m / z$ 666.2828 [M] $]^{+}$(calcd for $\mathrm{C}_{40} \mathrm{H}_{42} \mathrm{O}_{9}, 666.2830$ ).

Taiwaniacryptodimer B (2): gum; $[\alpha]_{\mathrm{D}}^{30}=-26.2$ (c 0.08, $\left.\mathrm{CHCl}_{3}\right)$; UV $(\mathrm{MeOH}): 229.0$ (4.32), 284.0 (4.06), 314.0 (3.53) nm; IR (Neat): 3576 (-OH), 1791 (ester C=O), 1616, 1499 (benzene ring) $\mathrm{cm}^{-1} ;{ }^{1} \mathrm{H}$ NMR $\left(500 \mathrm{MHz} \mathrm{CDCl}_{3}\right)$ : see Table $1 ;{ }^{13} \mathrm{C} \mathrm{NMR}\left(125 \mathrm{MHz}, \mathrm{CDCl}_{3}\right)$ : 
see Table 2); EIMS (70 eV) m/z (\%):666 ([M] $\left.]^{+}, 5\right), 368$ (8), 350 (98); HREIMS $m / z 666.2823$ $[\mathrm{M}]^{+}$(calcd for $\mathrm{C}_{40} \mathrm{H}_{42} \mathrm{O}_{9}, 666.2830$ ).

Taiwaniacryptodimer C (3): gum; $[\alpha]_{\mathrm{D}}^{30}=+105.4\left(c\right.$ 0.6, $\left.\mathrm{CHCl}_{3}\right)$; UV (MeOH): 227 (3.34), 284 (3.11), 309 (2.98) nm; IR (Neat): 1791 (ester C=O), 1618, 1498 (benzene ring) $\mathrm{cm}^{-1},{ }^{1} \mathrm{H}$ NMR (500 MHz, $\left.\mathrm{CDCl}_{3}\right)$ : see Table 1; ${ }^{13} \mathrm{C}$ NMR (125 MHz, $\left.\mathrm{CDCl}_{3}\right)$ : see Table 2); EIMS $(70 \mathrm{eV}) \mathrm{m} / z(\%): 666\left([\mathrm{M}]^{+}, 2\right), 350$ (95), 316 (100), 151 (76); HREIMS $m / z 666.2668[\mathrm{M}]^{+}$ (calcd for $\mathrm{C}_{40} \mathrm{H}_{40} \mathrm{O}_{9}, 666.2673$ ).

Taiwaniacryptodimer D (4): yellow gum; $[\alpha]_{\mathrm{D}}^{30}=-1.3\left(\right.$ ( $\left.0.71, \mathrm{CHCl}_{3}\right)$; $\mathrm{UV}(\mathrm{MeOH})$ : 214 (2.96), 234 (3.07) nm; IR (Neat): $3423(\mathrm{OH}), 1678$ (terminal double bond), 1615, 1464 (benzene ring) $\mathrm{cm}^{-1} ;{ }^{1} \mathrm{H}$ NMR (500 MHz, $\left.\mathrm{CDCl}_{3}\right)$ : see Table $1 ;{ }^{13} \mathrm{C} \mathrm{NMR}\left(125 \mathrm{MHz}, \mathrm{CDCl}_{3}\right)$ : see Table 2); EIMS (70 eV) m/z (\%): $518\left([\mathrm{M}]^{+}, 50\right), 202$ (100), 159 (79), 73 (52); HREIMS $m / z$ $518.3758[\mathrm{M}]^{+}$(calcd for $\left.\mathrm{C}_{35} \mathrm{H}_{50} \mathrm{O}_{3}, 518.3762\right)$.

Taiwaniacryptodimer E (5): yellow gum; $[\alpha]_{\mathrm{D}}^{30}=+19.6\left(c 0.39, \mathrm{CHCl}_{3}\right) ; \mathrm{UV}(\mathrm{MeOH})$ : 231 (4.10), 286 (3.42) nm; IR (Neat): $3453(\mathrm{OH}), 1712$ (ketone), 1464, 1421 (benzene ring) $\mathrm{cm}^{-1} ;{ }^{1} \mathrm{H}$ NMR (500 MHz, $\left.\mathrm{CDCl}_{3}\right)$ : see Table $\left.1 ;{ }^{13} \mathrm{C} \mathrm{NMR} \mathrm{(125} \mathrm{MHz,} \mathrm{CDCl}_{3}\right)$ : see Table 2); EIMS (70 eV) $m / z(\%): 534$ ([M] $\left.{ }^{+}, 85\right), 516$ (18), 220 (70), 205 (100), 202 (98); HREIMS $m / z$ $534.3703[\mathrm{M}]^{+}$(calcd for $\mathrm{C}_{35} \mathrm{H}_{50} \mathrm{O}_{4}, 534.3711$ ).

\subsection{Antifungal Activity Assays}

Test microorganisms. The in vitro antifungal activity of Compounds $\mathbf{1 - 5}$ were tested against a panel of laboratory control strains belonging to the Bioresource Collection and Research Center (BCRC), Hsinchu, Taiwan: fungal organisms Aspergillus niger (BCRC31512), Penicillium italicum (BCRC-30567), Candida albicans (BCRC-21538), and Saccharomyces cerevisiae (BCRC-20822).

\subsubsection{By Disc Diffusion Assay}

The antifungal susceptibility test of the isolated compounds was performed with the following strains: Aspergillus niger, Penicillium italicum, Candida albicans, and Saccharomyces cerevisiae by the disc diffusion method and applied following the CLSI M44-A, M44-S2 (for yeasts) [20,21], and M-51P (for filamentous fungi) guideline [22]. A standard disk of ketoconazole was used as a positive control, while the disk imbued with $50 \mu \mathrm{L}$ of pure DMSO was used as a negative control. The diameters of the inhibition zones were measured in millimeters and means of a slide caliper. Each test was performed in triplicate and repeated three times, and results were analyzed for statistical significance [20-22].

\subsubsection{By Broth Dilution Assay}

The MIC determination for the antifungal assay was performed according to the CLSI (Clinical and Laboratory Standard Institute) using the broth dilution assay methods [23-25]. Extract stock solutions and partitions were prepared in 5\% DMSO, and twofold serial dilutions were prepared in RPMI in 96-well microtiter plates (Corning Incorporated, Corning, NY, USA). The final concentrations ranged from 0.98 to $2.000 \mathrm{~g} \mathrm{~mL}^{-1}$. Test organisms $(100 \mu \mathrm{L})$ were added to each well in the microtiter plates. The growth control contained medium and inoculum. Blank controls contained medium only. The microtiter plates were then incubated at $35^{\circ} \mathrm{C}$ and the endpoints were read after $48 \mathrm{~h}$. The lowest concentration for each test compound at which color change occurred was recorded as its primary MIC value. The average of primary values from three individual tests were calculated, and that was taken as the final MIC value for each of the test compounds.

\section{Conclusions}

The research object of this research material Taiwania cryptomerioides (Taxodiaceae) is taxonomically included in one genus and one species of endemic plants in Taiwan. It contains more than $6 \%$ of essential oil in its heartwood. T. cryptomerioides is an important building material with high value in Taiwan. Previously, we investigated the chemical components of the heartwood and bark of this plant because of its antifungal and decayresistant 
characteristics as well as of its beautiful yellowish-red color with distinct purplish-pink streaks. The interesting compounds and those conferring biological activities isolated from the heartwood and bark of T. cryptomerioides prompted us to study the chemical components of its roots. In this study, we explored one novel constituent from the roots that had not been published in the past and evaluated and screened the metabolites for antifungal activity. It is found that the new dimer skeleton components $\mathbf{1}$ and $\mathbf{2}$ have moderate antifungal activity, one epoxide dimer 3 has weak activity, and two diterpenes 4 and 5 also possess moderate antifungal activities. The unique structure and antifungal activity of those have activities that make it an interesting material for further development.

Author Contributions: Y.-H.K. is responsible for the main research framework; Y.-H.K. and M.-J.C. designed the research; C.-F.C. performed the chemical and structure elucidation research; C.-L.C., M.D.W. and H.-S.C. conducted antifungal assays, Y.-H.K. and M.-J.C. helped with structure elucidation, Y.-H.K. and M.-J.C. organized the data and M.-J.C. wrote the paper. All authors have read and agreed to the published version of the manuscript.

Funding: This work was financially supported by China Medical University grant in Taiwan (10959189 and CMU109-AWARD-02) and "Chinese Medicine Research Center, China Medical University" from The Featured Areas Research Center Program within the framework of the Higher Education Sprout Project by the Ministry of Education (MOE) in Taiwan (CMRC-CHM-2-1). This work was partial kindly supported by the Food Industry Research and Development Institute (FIRDI) and supported by Ministry of Science and Technology, R.O.C. (MOST-108-2320-B-080-002- \& 1102320-B-080-001-).

Data Availability Statement: Not applicable.

Acknowledgments: The authors thank Senior Technician Chyi Jia Wang of Center for Resources, Research and Development (CRRD) of Kaohsiung Medical University for measuring the 2D NMR data.

Conflicts of Interest: The authors declare no conflict of interest.

\section{References}

1. Li, H.L.; Keng, H. Taxodiaceae. In Flora of Taiwan, 2nd ed.; Editorial Committee of the Flora of Taiwan: Taipei, Taiwan, 1994; Volume 1, pp. 582-585.

2. Cheng, Y.S.; Kuo, Y.H.; Lin, Y.T. Extractive components from the wood of Taiwania cryptomerioides Hayata: The structures of "T-cadinol" and "T-murrolol". J. Chem. Soc. Chem. Commun. 1967, 12, 565-566. [CrossRef]

3. Lin, Y.T.; Cheng, Y.S.; Kuo, Y.H. Extractive components from the wood of taiwania cryptomerioides hayata: A new sesquiterpene keto alcohol, cadinane-3-ene-9 $\alpha$-ol-2-one. Tetrahedron Lett. 1968, 9, 3881-3882. [CrossRef]

4. Kuo, Y.H.; Cheng, Y.S.; Lin, Y.T. Extractive components from the wood of taiwania cryptomerioides hayata: Three new sesquiterpene alcohols, muurolane-3-ene-9 $\beta$-ol-2-one, muurolane- $2 \delta, 9 \beta$-diol-3-ene, and muurolane-2 $\beta, 9 \beta$-diol-3-ene. Tetrahedron Lett. 1969, 10, 2375-2377. [CrossRef]

5. Kuo, Y.H.; Chang, C.I.; Lee, C.K. Six podocarpane-type trinorditerpenes from the bark of Taiwania cryptomerioides. Chem. Pharm. Bull. 2000, 48, 597-599. [CrossRef] [PubMed]

6. Kuo, Y.H.; Chang, C.I. Podocarpane-Type Trinorditerpenes from the Bark of Taiwania cryptomerioides. J. Nat. Prod. 2000, 63, 650-652. [CrossRef] [PubMed]

7. Kuo, Y.H.; Chien, S.C. Quinone-type podocarpanes from the bark of Taiwania cryptomerioides. Chem. Pharm. Bull. 2001, 49, 1033-1035. [CrossRef] [PubMed]

8. Kuo, Y.H.; Chien, S.C.; Huang, S.L. Four new podocarpane-type trinorditerpenes from the bark of Taiwania cryptomerioides. Chem. Pharm. Bull. 2002, 50, 544-546. [CrossRef] [PubMed]

9. Kuo, Y.H.; Chien, S.C.; Kuo, C.C. Cadinane-Type Sesquiterpenes from the Roots of Taiwania cryptomerioides HAYATA. Planta Med. 2002, 68, 1020-1023. [CrossRef] [PubMed]

10. Kuo, Y.H.; Chyu, C.F.; Lin, H.C.; Kuo, Y.H.; Chyu, C.F.; Lin, H.C. Cadinane-Type Sesquiterpenes from the Roots of Taiwania cryptomerioides Hayata. Chem. Pharm. Bull. 2003, 51, 986-989. [CrossRef] [PubMed]

11. Kuo, Y.H.; Chyu, C.F.; Kuo, Y.H.; Chyu, C.F. Two novel sesquiterpenes from the roots of Taiwania cryptomerioides Hayata. Tetrahedron Lett. 2003, 44, 7221-7223. [CrossRef]

12. Chyu, C.F.; Chiang, Y.M.; Lin, H.C.; Kuo, Y.H. Two novel 9,11-seco-11-norabietanes from the roots of Taiwania cryptomerioides. Tetrahedron Lett. 2004, 45, 641-643. [CrossRef]

13. Chyu, C.F.; Lin, H.C.; Kuo, Y.H. New abietane and seco-abietane diterpenes from the roots of Taiwania cryptomerioides. Chem. Pharm. Bull. 2005, 53, 11-14. [CrossRef] 
14. Chyu, C.F.; Ke, M.R.; Chang, Y.S.; Chien, S.C.; Kuo, Y.H. New cadinane-type sesquiterpenes from the roots of Taiwania cryptomerioides Hayata. Helv. Chim. Acta 2007, 8, 1514-1521. [CrossRef]

15. Gonzàlez, A.G.; Andrès, L.S.; Luis, J.G.; Brito, I.; Rodriguez, M.L. Diterpenes from Salvia mellifera. Phytochemistry 1991, 30 , 4067-4070. [CrossRef]

16. Lin, Y.T.; Wang, K.T.; Weinstein, B. Phytochemical studies. The structure of taiwanin A. J. Chem. Soc. Chem. Commun. 1965, 23, 592-593. [CrossRef]

17. Esquivel, B.; Flores, M.; Hernandez-Ortega, S.; Toscano, R.A.; Ramamoorthy, T.P. Abietane and icetexane diterpenoids from the roots of Salvia aspera. Phytochemistry 1995, 39, 139-143. [CrossRef]

18. Cao, W.; Deng, X.; Liu, M.; Liu, T.; Pan, Z.; Yang, S.; Zhou, Y. Efficient Synthesis of Icetexane Diterpenes and Apoptosis Inducing Effect by Upregulating BiP-ATF4-CHOP Axis in Colorectal Cells. J. Nat. Prod. 2021, 84, 2012-2019. [CrossRef] [PubMed]

19. San Feliciano, A.; Medarde, M.; Lopez, J.L.; Miguel del Corral, J.M.; Puebla, P.; Barrero, A.F. Terpenoids from leaves of Juniperus thurifera. Phytochemistry 1988, 27, 2241-2248. [CrossRef]

20. Clinical and Laboratory Standards Institute. Method for Antifungal Disk Diffusion Susceptibility Testing of Yeasts; Approved Guideline; CLSI document M44-A, Clinical and Laboratory Standards Institute: Wayne, PA, USA, 2004.

21. Clinical and Laboratory Standards Institute. Zone Diameter Interpretive Standards, Corresponding Minimal Inhibitory Concentration (MIC) Interpretive Breakpoints, and Quality Control Limits for Antifungal Disk Diffusion Susceptibility Testing of Yeasts, 2nd ed.; Informational supplement; CLSI document M44-S2, Clinical and Laboratory Standards Institute: Wayne, PA, USA, 2008.

22. Clinical and Laboratory Standards Institute. Method for Antifungal Disk Diffusion Susceptibility Testing of Filamentous Fungi; Proposed guideline; CLSI document M51-P, Clinical and Laboratory Standards Institute: Wayne, PA, USA, 2008.

23. Clinical and Laboratory Standards Institute. Reference Method for Broth Dilution Antifungal Susceptibility Testing of Yeasts, 3rd ed.; Approved standard; CLSI document M27-A3, Clinical and Laboratory Standards Institute: Wayne, PA, USA, 2008.

24. Clinical and Laboratory Standards Institute. Reference Method for Broth Dilution Antifungal Susceptibility Testing of Yeasts, 3rd ed.; Informational supplement; CLSI document M27-S3, Clinical and Laboratory Standards Institute: Wayne, PA, USA, 2008.

25. Clinical and Laboratory Standards Institute. Reference Method for Broth Dilution Antifungal Susceptibility Testing of Filamentous Fungi, 2nd ed.; Approved standard; CLSI document M38-A2, Clinical and Laboratory Standards Institute: Wayne, PA, USA, 2008. 